\title{
Magnetic Resonance Tractography and its Clinical Applications
}

Bahman Jalali Kondori", Elham Rahimian, Mohammad Hossein Asadi, Majid Reza Tahsini

Department of Anatomical Sciences, School of Medicine, Baqiyatallah University of Medical Sciences, Tehran, Iran.

\section{ABSTRACT}

Introduction: Magnetic resonance tractography is a method to identifying anatomical connections in the living human brain. This technique uses data obtained from diffusion of water molecules to display two-dimensional or three-dimensional images of the nerve fiber tracts through performing computational analysis. Thus, it will be possible to check the status of fibers with performing this technique. It is one of the advance techniques in the field of neuroscience which has a diagnostic potential in neurological diseases, such as brain tumors, normal pressure hydrocephalus, multiple sclerosis, stroke, and a large number of other neurological disorders. Conclusion: Tractography is a useful method to identify nerve tracts non-invasively and in-vivo. Compared to invasive techniques, non-invasive nature and ease of obtaining data enable this method to a suitable technique for pre-operative planning of brain surgery.

* Corresponding Author: Bahman Jalali Kondori

E-mail: Bahmanjalali2010@gmail.com 


\section{تراكتوكر افى تشديد مغناطيسى و كاربردهاى بالينى آن}

بهمن جلالى كندرى"، الهام رحيميان، محمد حسين اسدى، مجيد رضا تحسينى

كروه علوم تشريح، دانشكده يزشكى، دانشكاه علوم يزشكى بقيه الله، تهران، ايران.

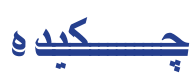

مقدمه: تراكتوگرافى تشديد مغناطيسى يك روش جهت شناشناسايى ارتباطات آناتوميك در مغز انسان

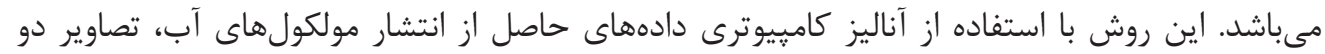

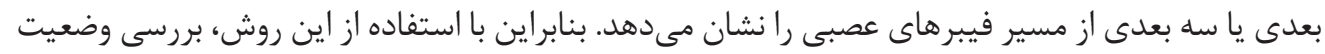

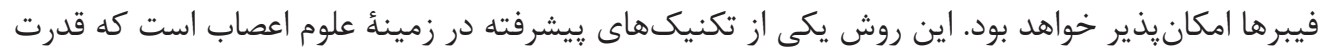

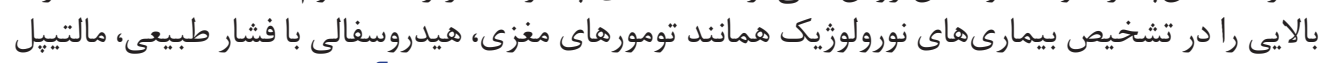

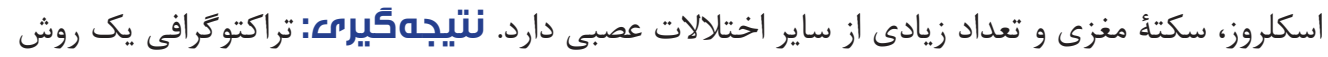

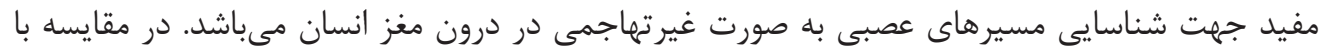

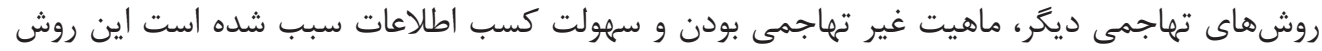

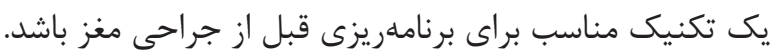




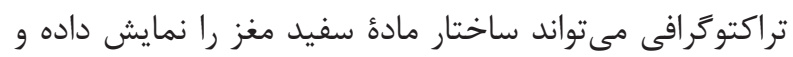

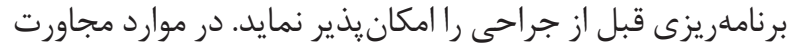

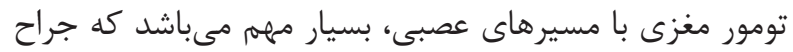

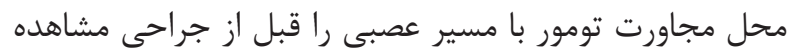

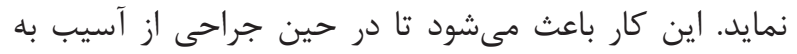

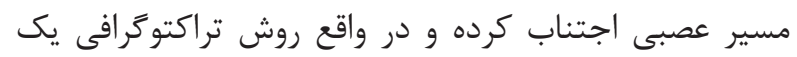

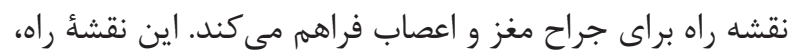

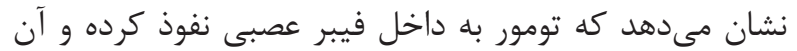
را تخريب كرده است يا فقط فيبر هاى عصبى ران كنار زده نده است.

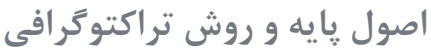

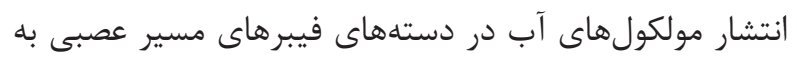

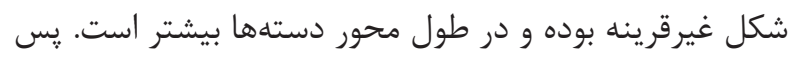

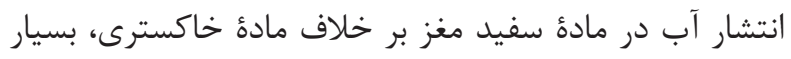

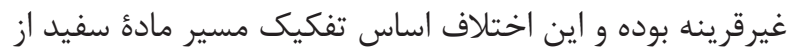

بافت خاكسترى سيستم اعصاب مركزى مى باشد (Y-Y (Y).

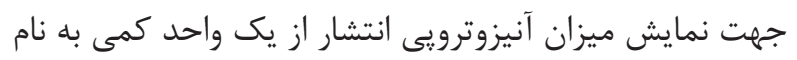

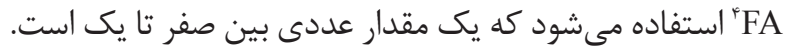

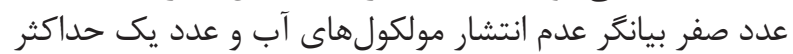

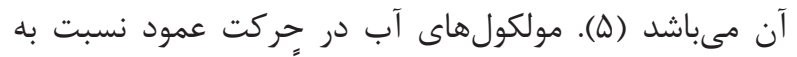

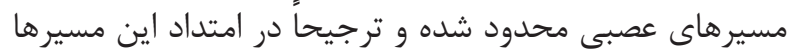

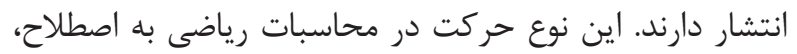
انتشار بيضى شكل ناميده مى شوند.

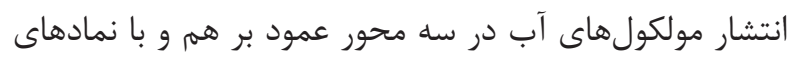

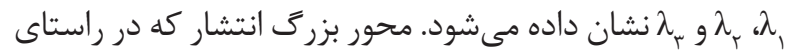

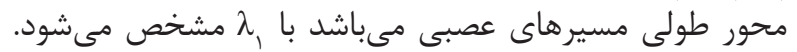

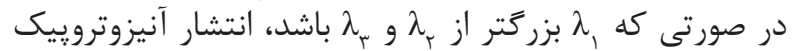
بوده و در صورتى كه كه زمانى كه انتشار در جهت محهور

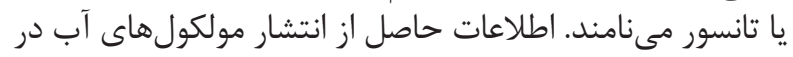

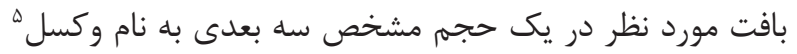

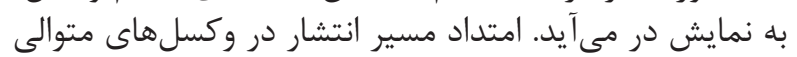

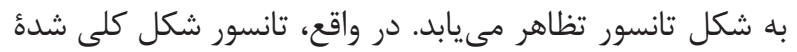

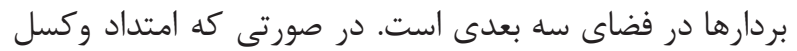

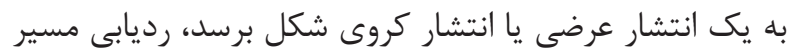

$$
\text { عصبى متوقف مىشود (9)-(تصوير (1). }
$$

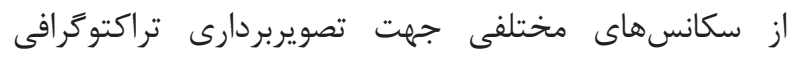
استفاده مىشود. در ابتدا از سكانسئهاى

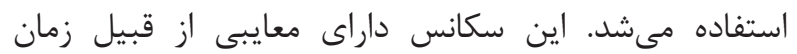

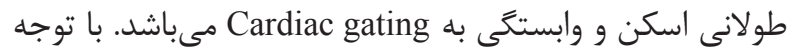

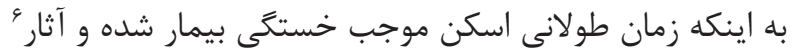

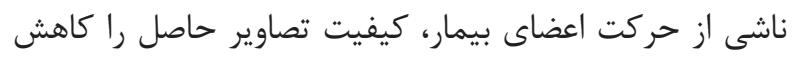

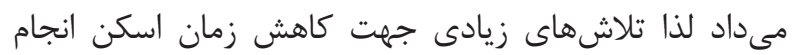

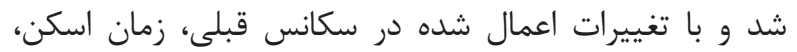

${ }^{1}$ Magnetic resonance imaging (MRI)

${ }^{2}$ Aaron Filler

${ }^{3}$ Peter Basser
تصويربردارى تشديد مغناطيسى (MRI) يك روش تصويربردارى

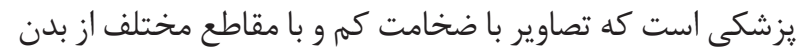

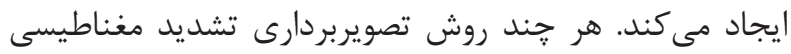

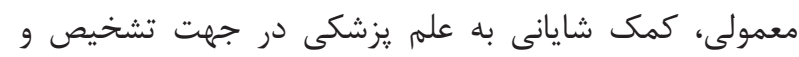

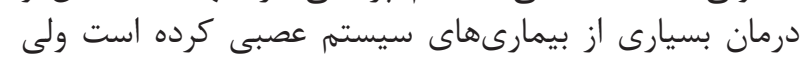
بررسى دقيق ماده سفيد مغز و مسيماري مسيرهاى عصبي سيتى به وسيلة اين

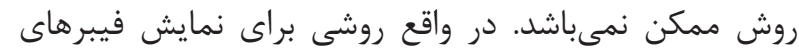

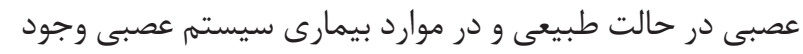

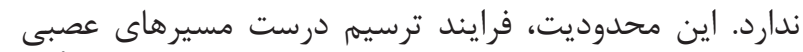

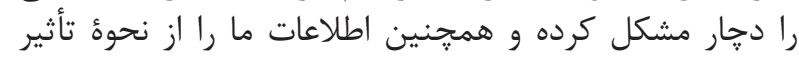

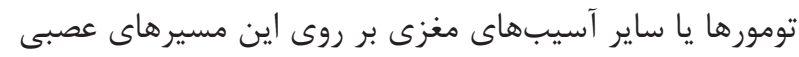
محدود كرده است.

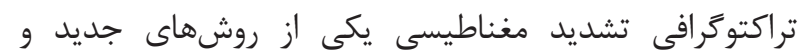

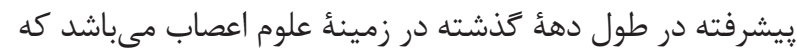

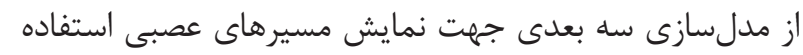

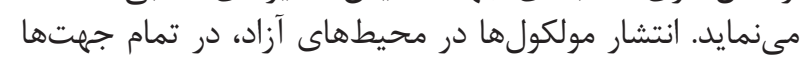

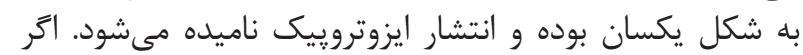

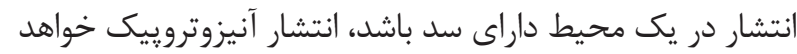

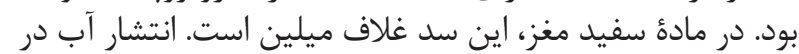

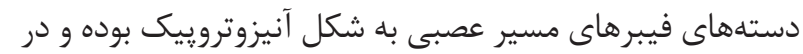

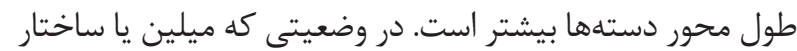

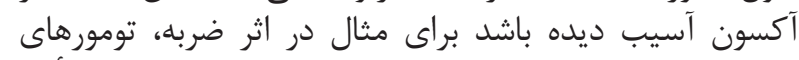

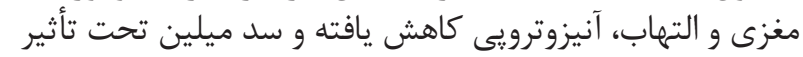

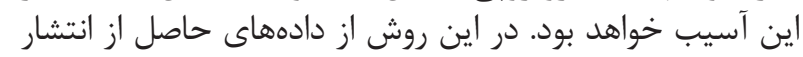

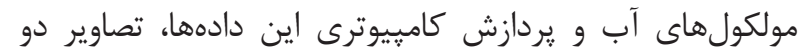

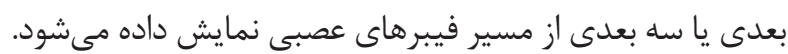
براى اولين بار در سال •99 199 محققى به نام ميشل موسلى

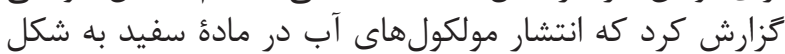

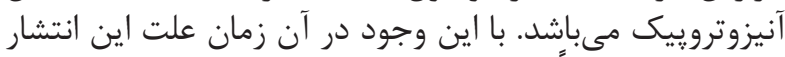

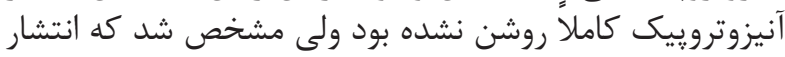

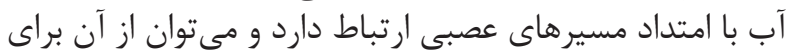

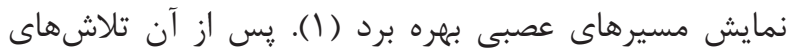

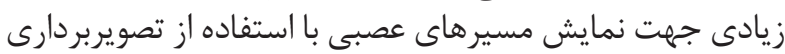

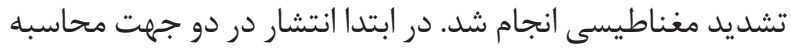

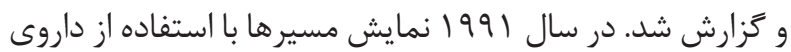

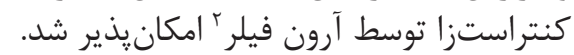

هند ماه بعد از آن، اولين تصوير مسيرهاى عصبى مغز بدون

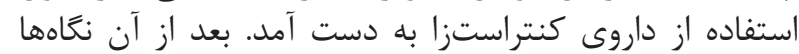

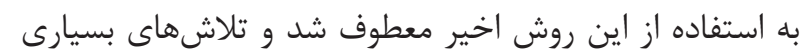

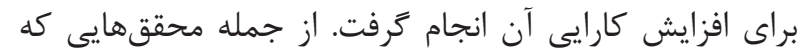

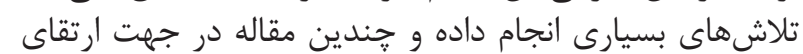

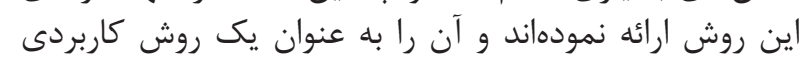

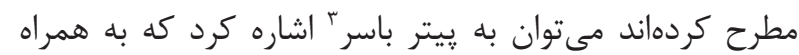

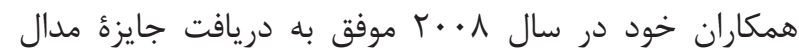

${ }^{4}$ Fractional Anisotropy (FA)

${ }^{5}$ Voxel

${ }^{6}$ Artifacts 


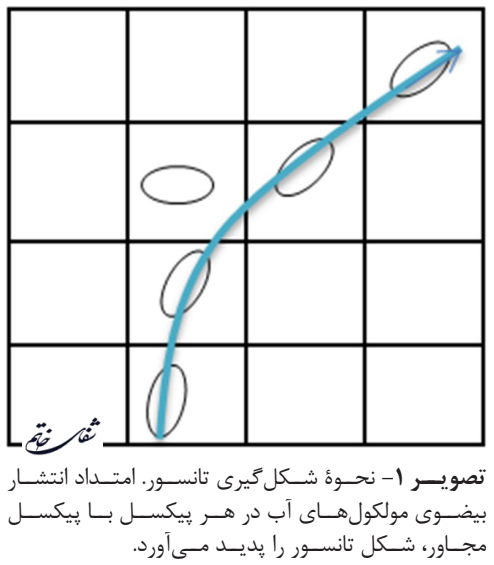

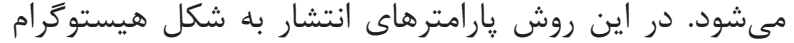

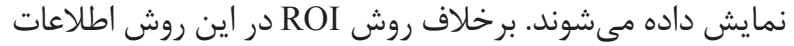

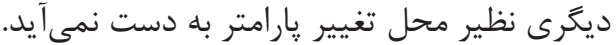

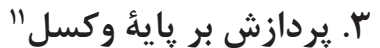
در سالهاى اخير اين روش بيشتر مورد توجه بوده است. در

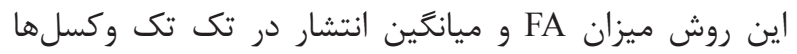

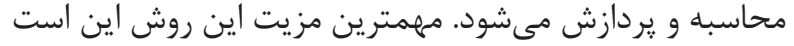

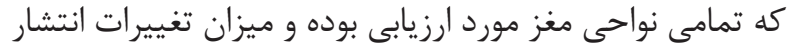

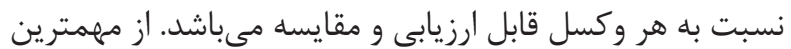

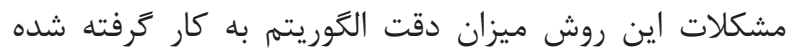
جهت محاسبه مى باشد.

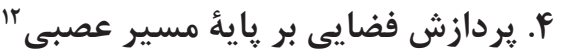

اين روش در واقع توسعه يافتهُ روش يردازش بر پايائ وكسل

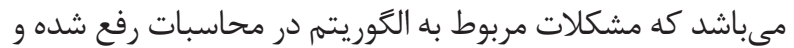

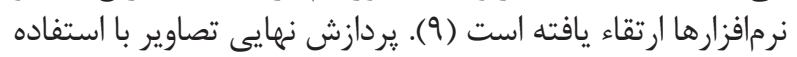

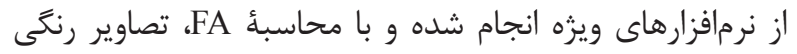

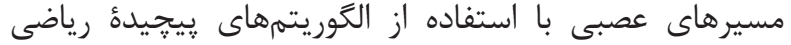

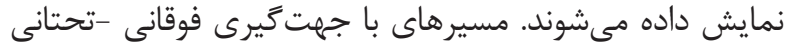

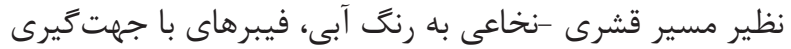

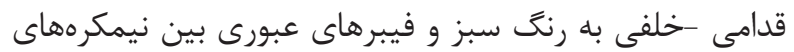

كاهش جشمخيرى داشت. اين سكانس جديد كه همجنان نيز

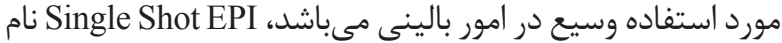

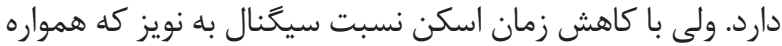

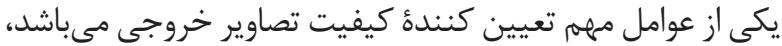

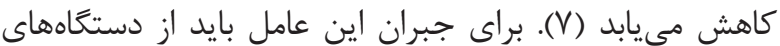

با قدرت ميدان مغناطيسى بالاتر استفاده شود (N).

علاوه بر سكانسهاى مختلف، روشهاى نمرئ نمونه

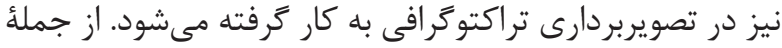

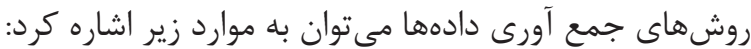

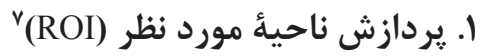

در بسيارى از تصويربردارىهاى بالينى تراكتوكرافى از اين روش إش

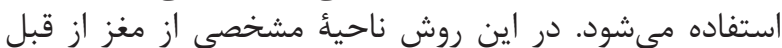

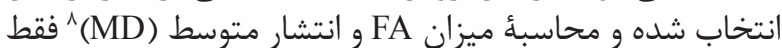

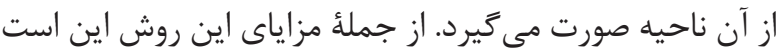
كه ناحئ مورد نظر مشخص مئ بوده و و تمركز

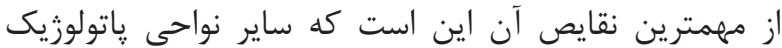

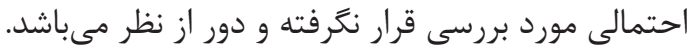

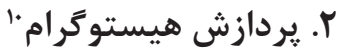
اين روش بيشتر در بيماران مبتلا به مولتييل اسكلروز يا تومور

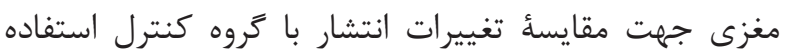

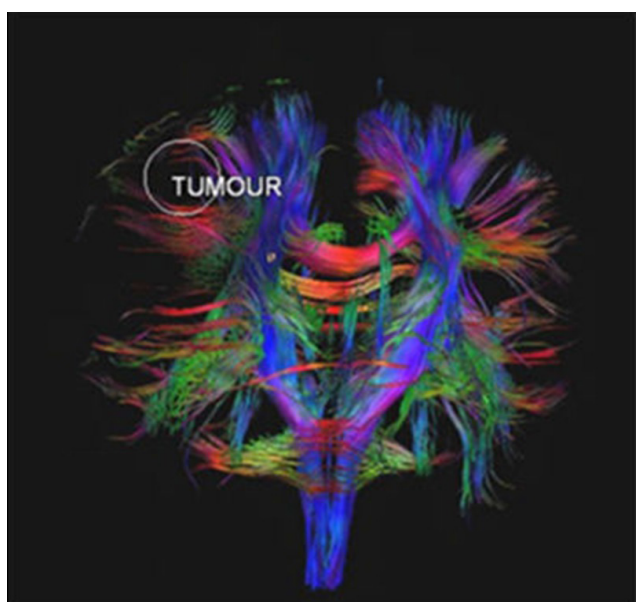

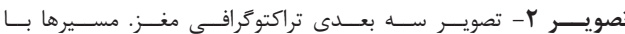

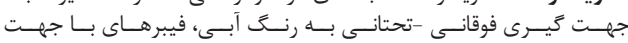

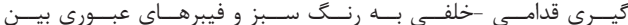

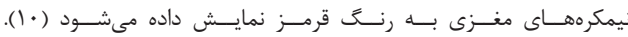




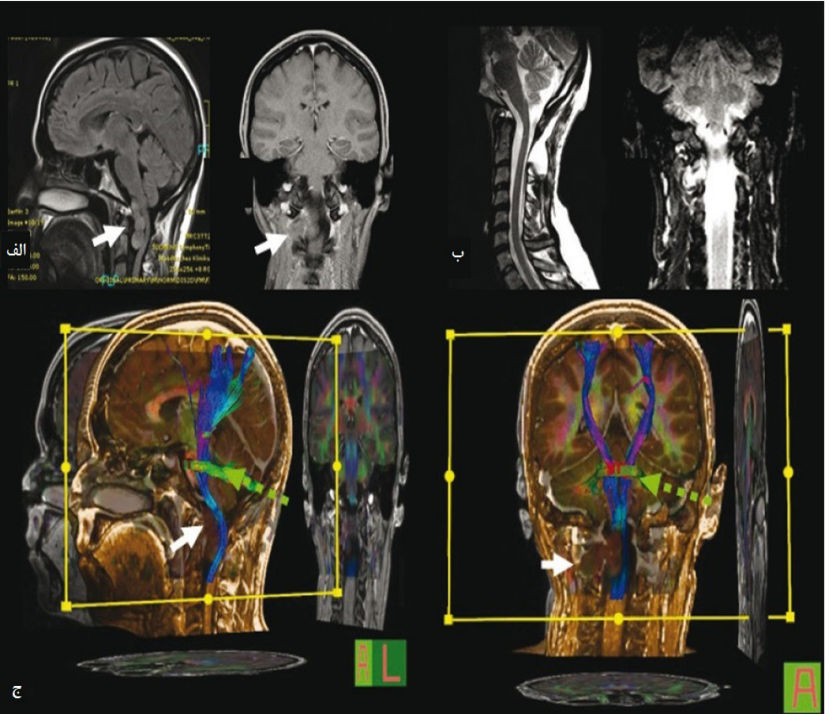

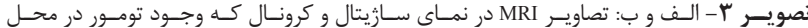

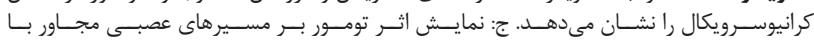

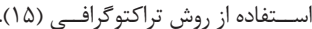

مىشود تا در حين جراحى از آسيب به مسير عصبى اجتناب

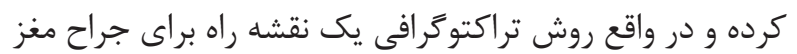

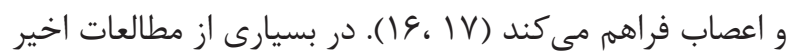

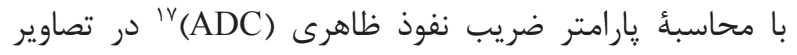

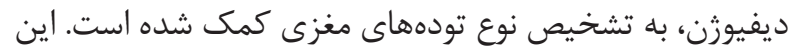

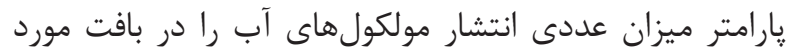

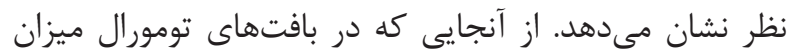

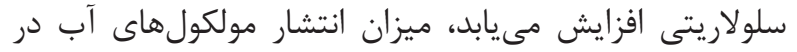

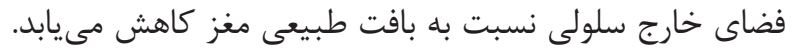

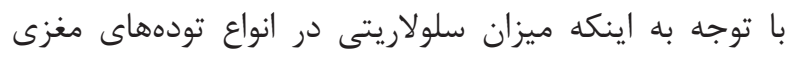

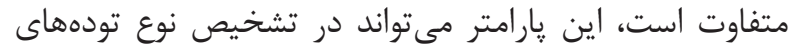

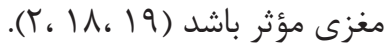
در مطالعاتى كه محققين در سالهاى اخير انجام دادهاند،

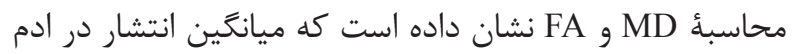

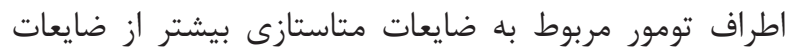

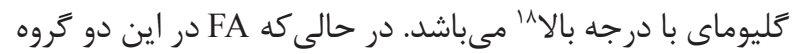

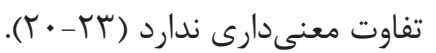

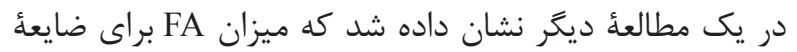

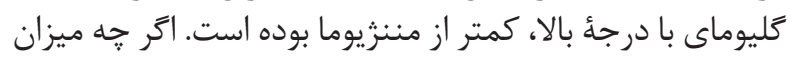

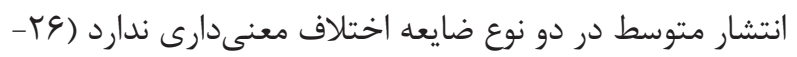
با TF

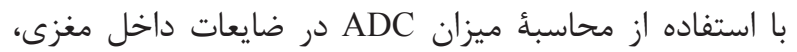

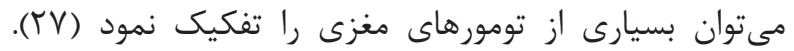

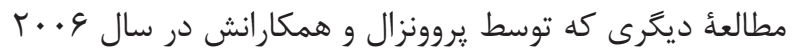

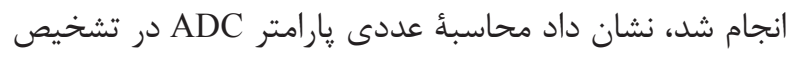
نوع تومورهاى مغزى مؤثر بوده است (Y) (Y).

\footnotetext{
${ }^{13}$ Normal pressure hydrocephalus

${ }^{14}$ Ischemic stroke

${ }^{15}$ Brain tumors
}

$$
\text { مغزى به رنغ قرمز خواهند بود (تصوير r). }
$$

با توجه به اينكه تصاوير سه بعدى حاصل، اطلاعات آناتوميك از بافت

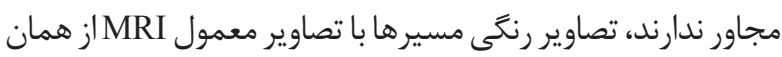

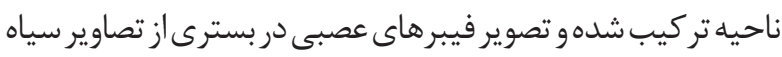

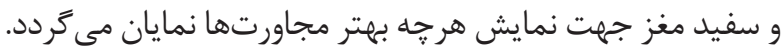

$$
\text { كاربردهاى بالينى تراكتوكر افى }
$$

اين روش قدرت تشخيصى بالايى در بيمارىهاى نورولوزيك إنيك

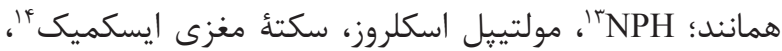

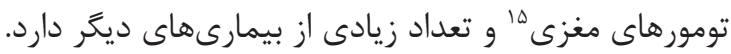

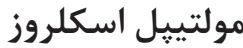

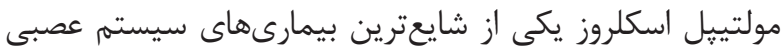

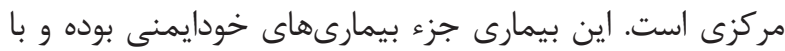

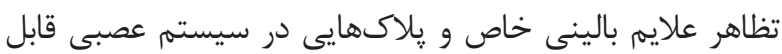

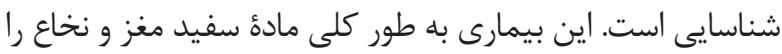

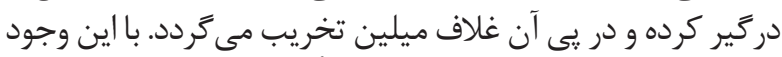

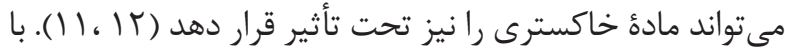

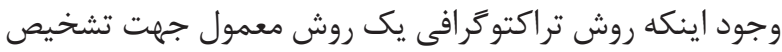

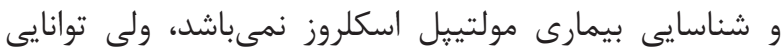

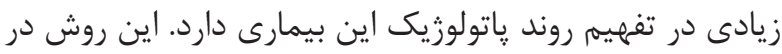

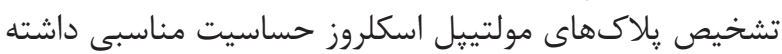

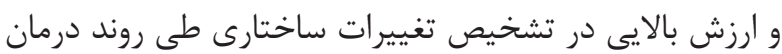

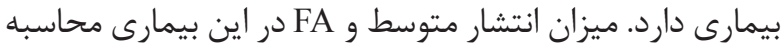

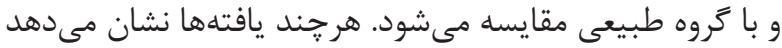

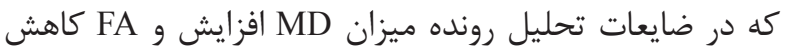

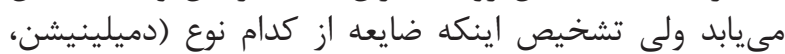

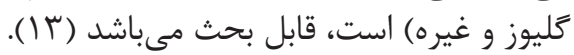

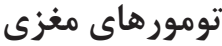

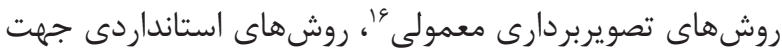

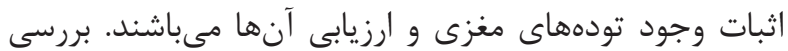

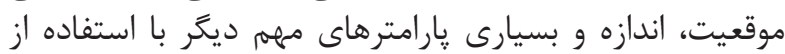

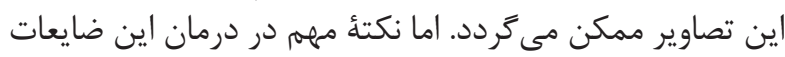

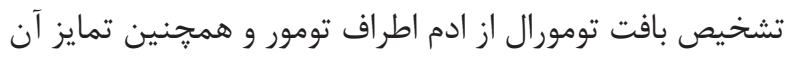
از مسيرهاى عصبى مجاور مى باشد.

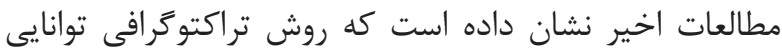

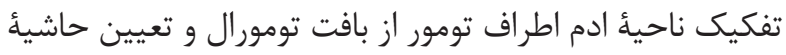

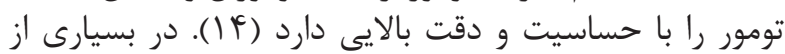

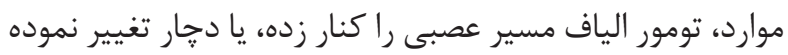

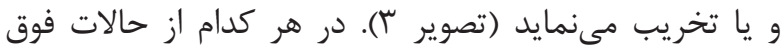

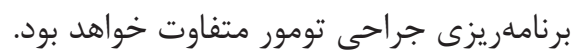

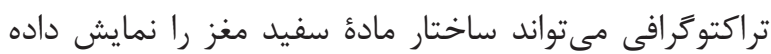

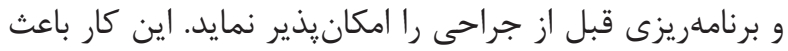

\footnotetext{
${ }^{16}$ Conventional

${ }^{17}$ Apparent diffusion coefficient (ADC)

${ }^{18}$ High-grade gliomas
} 


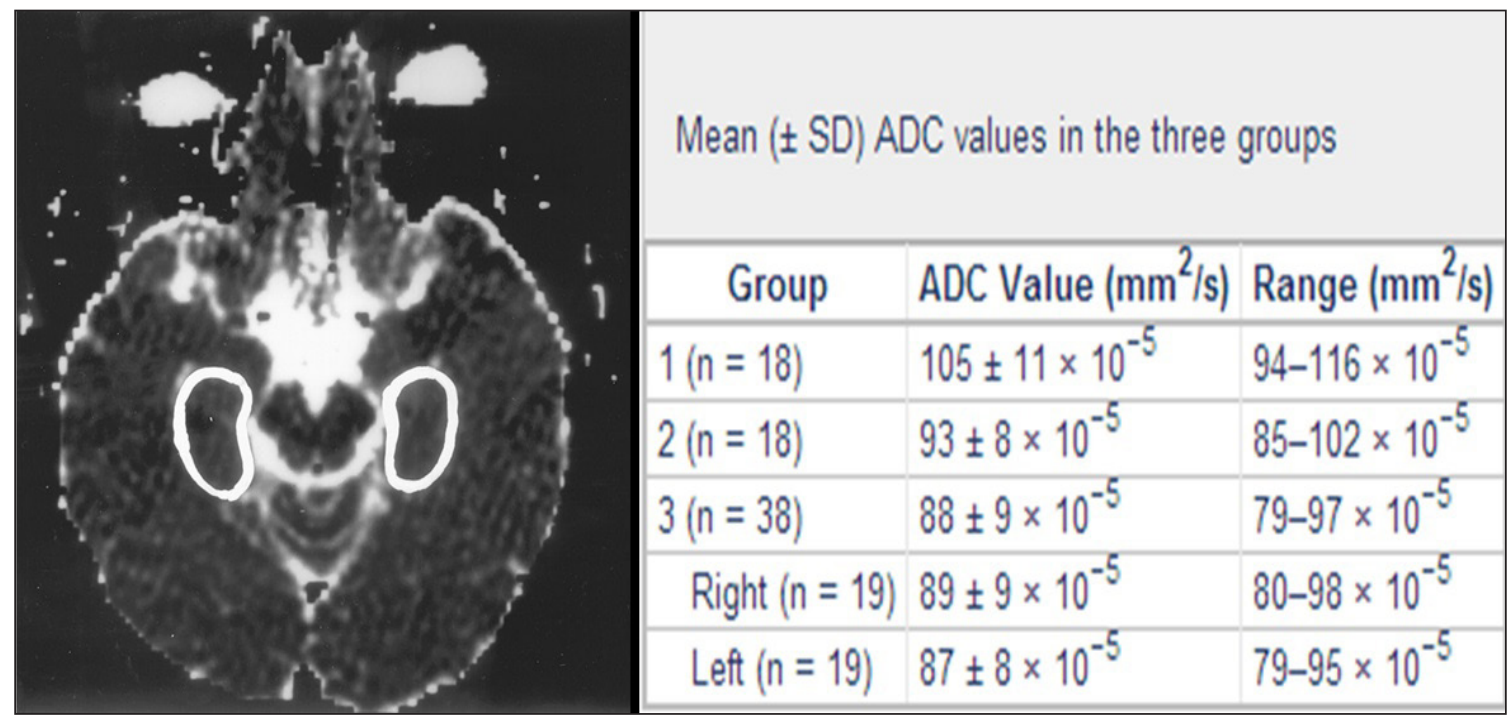

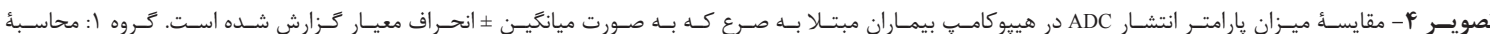

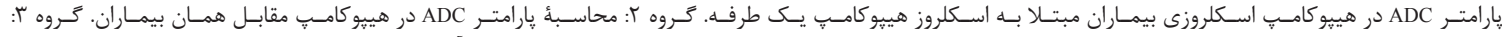

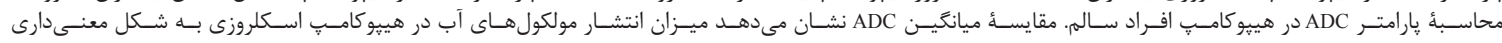

مىتوان اختلال در اين مسير را مورد بررسى قرار داد (ه؟).

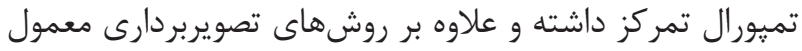

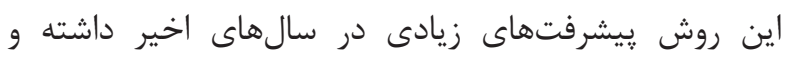

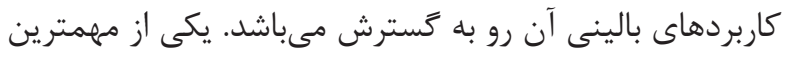

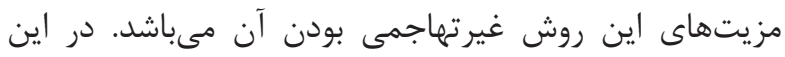

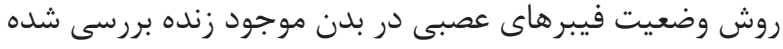

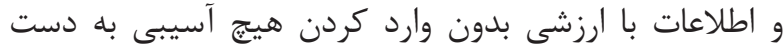

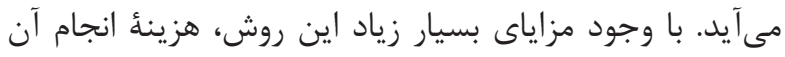

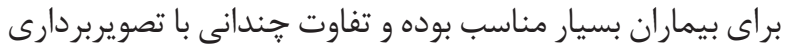

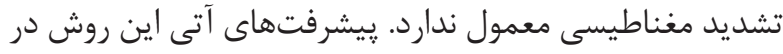

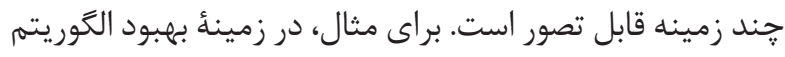

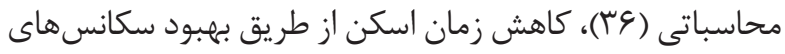

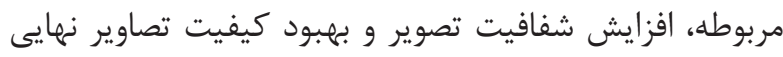

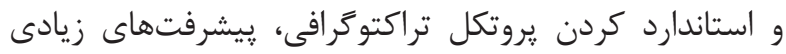
مى توان تصور نمود.

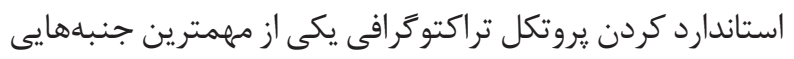

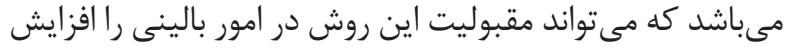

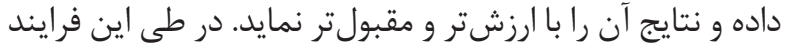

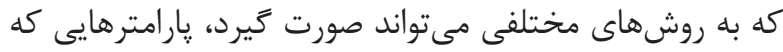

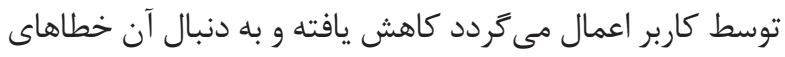

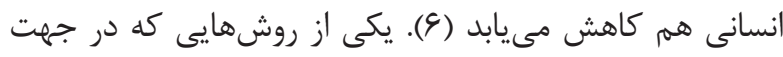

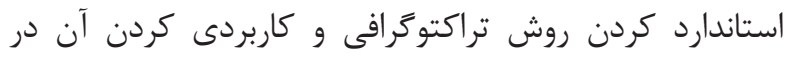

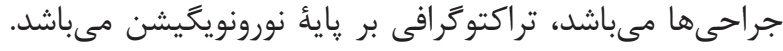

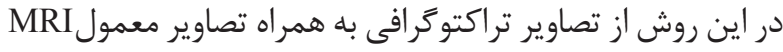

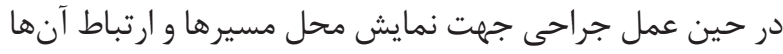

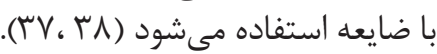

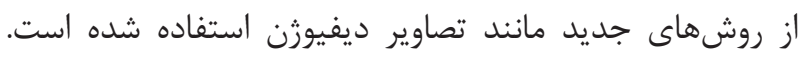

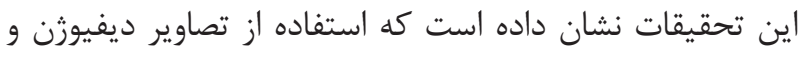

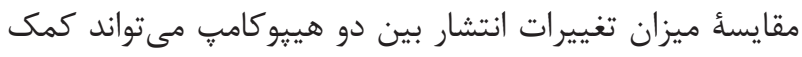

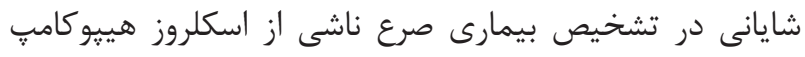

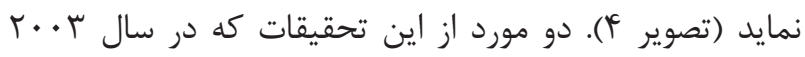

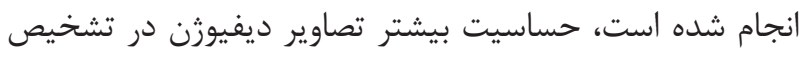

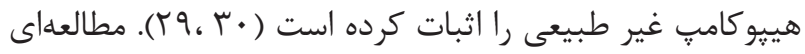

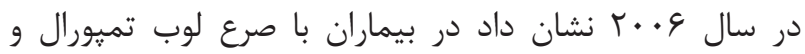

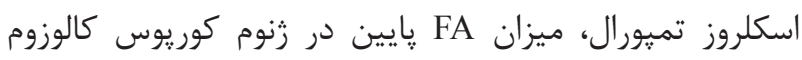

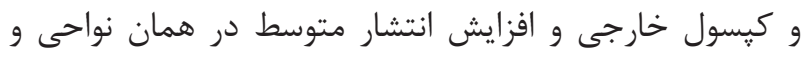

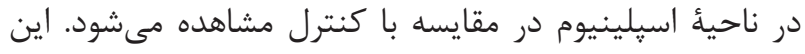

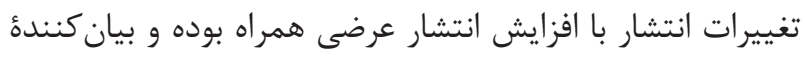

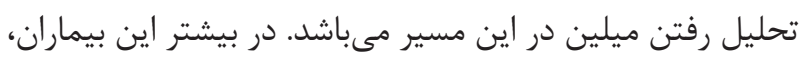

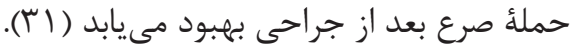

\section{سكتؤ مغزى ايسكميك}

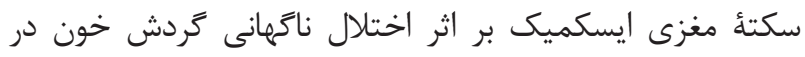

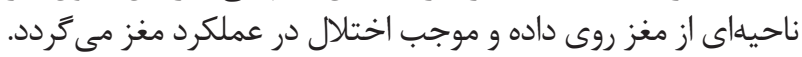

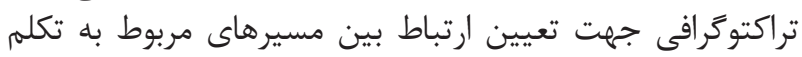

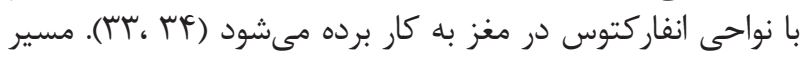

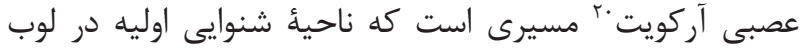

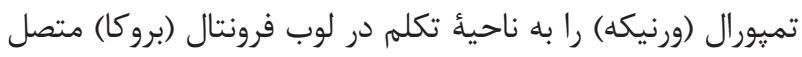

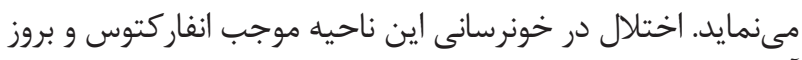

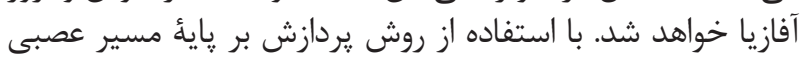


1. Moseley ME, Cohen Y, Kucharczyk J, Mintorovitch J, Asgari HS, Wendland MF. Diffusion-weighted MR imaging of anisotropic water diffusion in cat central nervous system. Radiology. 1990; 176 (2): 439-45.

2. Le Bihan D, Breton E, Lallemand D, Grenier P, Cabanis E, Laval-Jeantet M. MR imaging of intravoxel incoherent motions: application to diffusion and perfusion in neurologic disorders. Radiology. 1986; 161: 401-7.

3. Mori S, Barker PB. Diffusion magnetic resonance imaging: its principle and applications. Anat Rec. 1999; 257: 102-9.

4. van Gelderen P, de Vleeschouwer MH, DesPres D, Pekar J, van Zijl PC, Moonen CT. Water diffusion and acute stroke. Magn Reson Med. 1994; 31: 154-63.

5. Le Bihan D, Mangin JF, Poupon C, Clark CA, Pappata S, Molko N, et al . Diffusion tensor imaging: concepts and applications . J Magn Reson Imaging. 2001; 13(4): 534-46.

6. Yamada K, Sakai K, Akazawa K, Yuen S, Nishimura T. MR tractography: a review of its clinical applications. Magn Reson Med Sci. 2009; 8(4): 165-74.

7. Yamada K, Kizu O, Mori S, Ito H, Nakamura H, Yuen $\mathrm{S}$, et al. Brain fiber tracking with clinically feasible diffusion-tensor MR imaging: initial experience. Radiology. 2003; 227: 295-301.

8. Okada T, Miki Y, Fushimi Y, Hanakawa T, Kanagaki M, Yamamoto A, et al. Diffusion-tensor fiber tractography: intraindividual comparison of 3.0-T and 1.5-T MR imaging. Radiology. 2006; 238: 668-78.

9. Berg HJ, E.J. Behrens T. Diffusion MRI: from quantitative measurement to in-vivo neuroanatomy. 1st ed. Academic Press. 2009.

10. Zamzuri I, W M Nazaruddin W H, Muzaimi M, Badrisyah I, Rahman Izaini G, Jafri Malin A. Functional MRI, diffusion tensor imaging, magnetic source imaging and intraoperative neuromonitoring guided brain tumor resection in awake and under general anaesthesia. Lichtor T. Clinical management and evolving novel therapeutic strategies for patients with brain tumors. Online Book. InTech. 2013; p. 19.

11. Bjartmar C, Trapp BD. Axonal and neuronal degeneration in multiple sclerosis: mechanisms and functional consequences. Curr Opin Neurol. 2001; 14: 271-8.

12. Baum HM, Rothschild BB. The incidence and prevalence of reported multiple sclerosis. Ann Neurol. $1981 ; 10(5): 420-8$.

13. Castriota-Scanderbeg A, Sabatini U, Fasano F, Floris R, Fraracci L, Mario MD, et al. Diffusion of water in large demyelinating lesions: a follow-up study. Neuroradiology. 2002; 44: 764-7.

14. Price SJ, Jena R, Burnet NG, Hutchinson PJ, Dean AF, Pena A, et al. Improved delineation of glioma margins and regions of infiltration with the use of diffusion tensor imaging: an image guided biopsy study. AJNR Am J Neuroradiol. 2006; 27: 1969-74.

15. Carvi Y Nievas MN1, Hoellerhage HG, Drahten C. White matter tract alterations assessed with diffusion tensor imaging and tractography in patients with solid posterior fossa tumors. Neurol India. 2010; 58(6):914-21.

16. Iliescu B, Dabija A, Faiyad Z, Gavas S, Poeată I. The role of MR tractography in pre-surgical planningpersonal series of 25 cases and a review of the literature. Rom Neurosurg. 2011; 4: 483-90.

17. Bittar RG, Olivier A, Sadikot AF, Andermann F, Reutens DC. Cortical motor and somatosensory representation: effect of cerebral lesions. J Neurosurg. 2000; 92(2): 242-8.

18. Okamoto K, Ito J, Ishikawa K, Sakai K, Tokiguchi S. Diffusion-weighted echo-planar MR imaging in differential diagnosis of brain tumors and tumor-like conditions. Eur Radiol. 2000; 10: 1342-50.

19. Guo AC, Cummings TJ, Dash RC, Provenzale JM. Lymphomas and high-grade astrocytomas: comparison of water diffusibility and histologic characteristics. Radiology. 2002; 224(1): 177-83.

20. Lu S, Ahn D, Johnson G, Cha S. Peritumoral diffusion tensor imaging of high-grade gliomas and metastatic brain tumors. AJNR Am J Neuroradiol. 2003; 24: 937-41.

21. Tsuchiya K, Fujikawa A, Nakajima M, Honya K. Differentiation between solitary brain metastasis and high-grade glioma by diffusion tensor imaging. $\mathrm{Br} \mathrm{J}$ Radiol. 2005; 78: 533-7.

22. Chiang IC, Kuo YT, Lu CY, Yeung KW, Lin WC, Sheu FO, et al. Distinction between high-grade gliomas and solitary metastases using peritumoral 3-T magnetic resonance spectroscopy, diffusion, and perfusion imagings. Neuroradiology. 2004; 46: 619-27.

23. Krabbe K, Gideon P, Wagn P, Hansen U, Thomsen C, Madsen F. MR diffusion imaging of human intracranial 


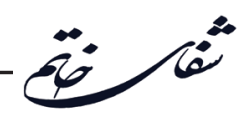

tumours. Neuroradiology. 1997; 39: 483-9.

24. Provenzale JM, McGraw P, Mhatre P, Guo AC, Delong D. Peritumoral brain regions in gliomas and meningiomas: investigation with isotropic diffusionweighted MR imaging and diffusion- tensor MR imaging. Radiology. 2004; 232: 451-60.

25. Bastin ME, Sinha S, Whittle IR, Wardlaw JM. Measurements of water diffusion and $\mathrm{T} 1$ values in peritumoural oedematous brain. Neuroreport. 2002; 13: 1335-40.

26. Kono K, Inoue Y, Nakayama K, Shakudo M, Morino $\mathrm{M}$, Ohata $\mathrm{K}$, et al. The role of diffusion-weighted imaging in patients with brain tumors. AJNR Am J Neuroradiol. 2001; 22: 1081-8.

27. Stadnik TW, Chaskis C, Michotte A, Shabana WM, van Rompaey K, Luypaert R, et al. Diffusion-weighted MR imaging of intracerebral masses: comparison with conventional MR imaging and histologic findings. AJNR Am J Neuroradiol. 2001; 22: 969-76.

28. Provenzale JM, Mukundan S, Barboriak DP. Diffusion-weighted and perfusion MR imaging for brain tumor characterization and assessment of treatment response. Radiology. 2006; 239(3): 632-49.

29. Assaf BA, Mohamed FB, bou-Khaled KJ, Williams JM, Yazeji MS, Haselgrove J, et al. Diffusion tensor imaging of the hippocampal formation in temporal lobe epilepsy. AJNR Am J Neuroradiol. 2003; 24: 1857-62.

30. Londono A, Castillo M, Lee YZ, Smith JK. Apparent diffusion coefficient measurements in the hippocampi in patients with temporal lobe seizures. AJNR Am J Neuroradiol. 2003; 24: 1582-6.

31. Gross DW, Concha L, Beaulieu C. Extratemporal white matter abnormalities in mesial temporal lobe epilepsy demonstrated with diffusion tensor imaging. Epilepsia. 2006; 47: 1360-3.

32. Wehner T, Lapresto E, Tkach J, Liu P, Bingaman W, Prayson RA, et al. The value of interictal diffusionweighted imaging in lateralizing temporal lobe epilepsy. Neurology. 2007; 68: 122-7.

33. Yamada K, Mori S, Nakamura H, Ito H, Kizu $\mathrm{O}$, Shiga K, et al. Fiber-Tracking method reveals sensorimotor pathway involvement in stroke patients. Stroke. 2003; 34(9): E159-62.

34. Kunimatsu A, Aoki S, Masutani Y, Abe O, Mori H, Ohtomo K. Three dimensional white matter tractography by diffusion tensor imaging in ischemic stroke involving the corticospinal tract. Neuroradiology. 2003; 45(8): 532-5.

35. Yamada K, Nagakane T, Mizuno T, Hosomi A, Nakagawa M, Nishimura T. MR Tractography depicting damage to the Arcuate fasciculus in a patient with conduction aphasia. Neurology. 2007; 68(10): 789.

36. Rowe M, Zhang HG, Oxtoby N, Alexander DC. Beyond crossing fibers: Tractography exploiting subvoxel fibre dispersion and neighbourhood structure. Inf Process Med Imaging. 2013; 7917: 402-13.

37. Coenen VA, Krings T, Mayfrank L, Polin RS, Reinges $\mathrm{MH}$, Thron A, et al. Three-dimensional visualization of the pyramidal tract in a neuronavigation system during brain tumor surgery: first experiences and technical note. Neurosurgery. 2001; 49: 86-92; discussion 92-3.

38. Krieg SM, Buchmann NH, Gempt J, Shiban E, Meyer B, Ringel F. Diffusion tensor imaging fiber tracking using navigated brain stimulation a feasibility study. Acta Neurochir. 2012; 154(3): 555-63. 